\title{
Seleção de tecnologias de comunicações no exército brasileiro utilizando os métodos multicritério de análise hierárquica, TODIM e software Sapiens
}

\author{
Livia de Souza Ribeiro ${ }^{\mathrm{a} *}$, Aderson Campos Passos ${ }^{\mathrm{b}}$, \\ Marcello Goulart Teixeira ${ }^{\mathrm{c}}$ \\ a*liviaribeiro14@gmail.com, IME, Brasil \\ baderson@ime.eb.br, IME, Brasil \\ 'marcellogt@dcc.ufrj.br, UFRJ, Brasil
}

\begin{abstract}
Resumo
No Exército Brasileiro, as redes de comunicações apresentam características especiais. Um sistema tático de comunicação deve ser uma única estrutura lógica que integra múltiplas tecnologias, como os Subsistemas de Rádio de Combate e de Dados. Este trabalho mostra um estudo de caso para a seleção de tecnologia que atenda aos critérios descritos pelo Exército. Para priorizar seis alternativas, são aplicados dois métodos que auxiliam a tomada de decisão: o método AHP Clássico e o TODIM. No estudo com o AHP, é mostrado cada passo da metodologia até a conclusão do resultado. Com o TODIM, é usado um software de apoio ao uso do método, o Sapiens. Foram realizadas entrevistas com especialistas da área de comunicações para o levantamento das informações necessárias. Considera-se que o principal resultado desse trabalho é a construção de um processo estruturado para essa seleção e não a obtenção dos resultados em si.
\end{abstract}

Palavras-chave

Análise de decisão multicritério. TODIM. AHP. Comunicações militares. Software Sapiens.

\section{Introdução}

Em um panorama militar, as redes de comunicações apresentam características especiais que as diferenciam das demais. Elas precisam estar adequadas ao ambiente em que serão aplicadas, de forma que possibilitem o comando e controle das ordens enviadas do escalão superior para o inferior, isto é, uma organização hierárquica de toda força militar (SALLES et al., 2008).

Exércitos de vários lugares do mundo dispõem de modernos sistemas táticos de Comando e Controle aplicados em situações de combate convencional. Esses sistemas podem ser empregados no sistema de comunicações do Exército Brasileiro, desde que atendam aos critérios operacionais descritos em manuais de campanha do Exército.

O Sistema de Comando e Controle é um sistema auxiliar do comandante, com a finalidade de apoiar e facilitar a tomada de decisões (BRASIL, 1995).
Com tal propósito, o Exército Brasileiro estrutura o Sistema de Comunicações do Exército (SICOMEx) em Sistema Estratégico de Comunicações (SEC) e Sistema Tático de Comunicações (SISTAC), (BRASIL, 1998). Além disso, o modelo utilizado permite a ligação com o Sistema Nacional de Telecomunicações e com os demais sistemas governamentais.

O SEC tem por objetivo o estabelecimento das ligações de longa distância, dentro do território nacional, para o atendimento das necessidades correntes e estratégicas do Exército, podendo, excepcionalmente, prestar apoio de comunicações a qualquer escalão do Exército Brasileiro no exterior. Para tanto, este sistema dispõe de meios de comunicações de grande versatilidade para o estabelecimento das ligações, como a Rede Corporativa Privativa do Exército (EBNet), as Redes Rádio do Sistema Estratégico de Comunicações (RRSEC) e a Rede Integrada de 
Telecomunicações do Exército (RITEx). Além destes, permite a integração com sistemas de comunicações do Ministério da Defesa e de outros Ministérios, como o Sistema de Comunicações Militares por Satélite (SISCOMIS) e o Sistema de Vigilância da Amazônia (SIVAM).

Já o SISTAC é o conjunto de meios de comunicações e informática pertencente a unidades operacionais do Exército Brasileiro, destinado ao preparo e emprego de tropas. Sua utilização, portanto, se dá em missões de adestramento ou em operações de campanha. Este sistema, que é o foco do presente trabalho, está subdividido em Sistema de Comunicações de área (SCA) e Sistema de Comunicações de Comando (SCC).

Esse trabalho mostra a construção de quatro modelos multicritério para resolver dois problemas de seleção de equipamentos para comunicação militar. Os dois problemas estão relacionados à seleção da tecnologia mais adequada para o Subsistema de Rádio de Combate e para o Subsistema de Rádio de Dados. Ambos compõem a estrutura de comunicações táticas de campanha do Exército Brasileiro. Para cada um desses processos de escolha, foram utilizados dois métodos multicritério: o TODIM e o Método de Análise Hierárquica Clássico (AHP). Será feita uma breve revisão de ambos os métodos e, posteriormente, será desenvolvido o estudo de caso mostrando como os critérios de decisão foram estruturados e os resultados obtidos. Os julgamentos utilizados foram emitidos por oficiais engenheiros militares do Exército Brasileiro: dois professores e dois alunos de pós-graduação do Instituto Militar de Engenharia (IME). Estes oficiais, além de possuírem sólida formação acadêmica, possuem vivência prática com os sistemas táticos citados.

Um resultado importante mostrado nesse texto é o desenvolvimento do Software Sapiens, que implementa o método TODIM. Aqui, o referido método é implementado totalmente com a utilização desse software, que é gratuito e de muito fácil utilização.

Ao fim, os resultados obtidos por ambos os métodos são comparados e analisados. É importante ressaltar que o objetivo desse texto não é a definição da melhor tecnologia para cada tipo de subsistema e sim discutir uma forma adequada para abordar esse problema, que é complexo e exige informações técnicas e a opinião de especialistas com conhecimento e vivência sobre o assunto.

\section{0 método de análise hierárquica clássico}

0 Método da Análise Hierárquica foi desenvolvido na década de 70 por Thomas L. Saaty. Em inglês, seu nome original é Analytic Hierarchy Process (AHP). 0 método leva esse nome por organizar os critérios de forma hierárquica na qual o problema decisório assume a posição do topo da hierarquia. Das inúmeras referências existentes na literatura sobre o AHP, recomenda-se a leitura dos livros escritos pelo próprio professor Saaty. Um desses livros é Saaty (2006), referenciado no final do artigo. Nele são mostrados detalhes do AHP, aplicações, exemplos detalhados e claros e os diversos livros já escritos sobre o método.

Após a escolha de critérios-chave para a tomada de decisão, outros subcritérios são escolhidos de maneira a especificar melhor esse conjunto de considerações, facilitar a sua valorização e assim sucessivamente, tomando a forma de uma árvore, conforme mostrado na Figura 1.

O AHP assume 3 considerações:

- É possível estabelecer uma preferência entre cada alternativa, baseado em cada critério separadamente, de forma independente e com resultados numéricos;

- A valorização de cada critério pode ser calculada a partir de seus subcritérios, na forma de uma árvore;

- No nível mais baixo da hierarquia (folhas da árvore), os subcritérios podem ser valorados a partir de comparações paritárias.

Ao se comparar duas alternativas sobre a óptica de determinado subcritério, devemos atribuir um valor numérico que representa a superioridade de uma alternativa sobre a outra nessa comparação. Quando lidamos com critérios quantitativos devemos levar em conta a ideia de função de utilidade antes de fazermos nossas comparações.

0 método AHP determina 9 níveis para a comparação entre critérios. A existência de um limite superior mostrou-se útil para minimizar o problema da superestimação de valores quando as disparidades são muito elevadas. Foram escolhidos 9 níveis devido à inerente facilidade humana em avaliar um item através da tricotomia: rejeição, indiferença e aceitação, e da facilidade de subdividir cada um desses itens em três níveis: baixo, médio e alto. A Tabela 1 mostra a escala fundamental de Saaty, na qual estão explícitos os nove níveis de julgamentos citados.

Com base nessas comparações é montada uma matriz quadrada para cada subcritério, na qual os valores de cada elemento da matriz são valores obtidos pela comparação das alternativas paritariamente. 0 autovetor normalizado da matriz determina a valoração de cada alternativa sob a luz de cada critério. Uma forma alternativa para calcular o vetor de pesos da matriz de comparações paritárias é definir esse vetor como a normalização da média aritmética das linhas dessa matriz, com essa normalização feita através da divisão do vetor pela soma de seus elementos. 


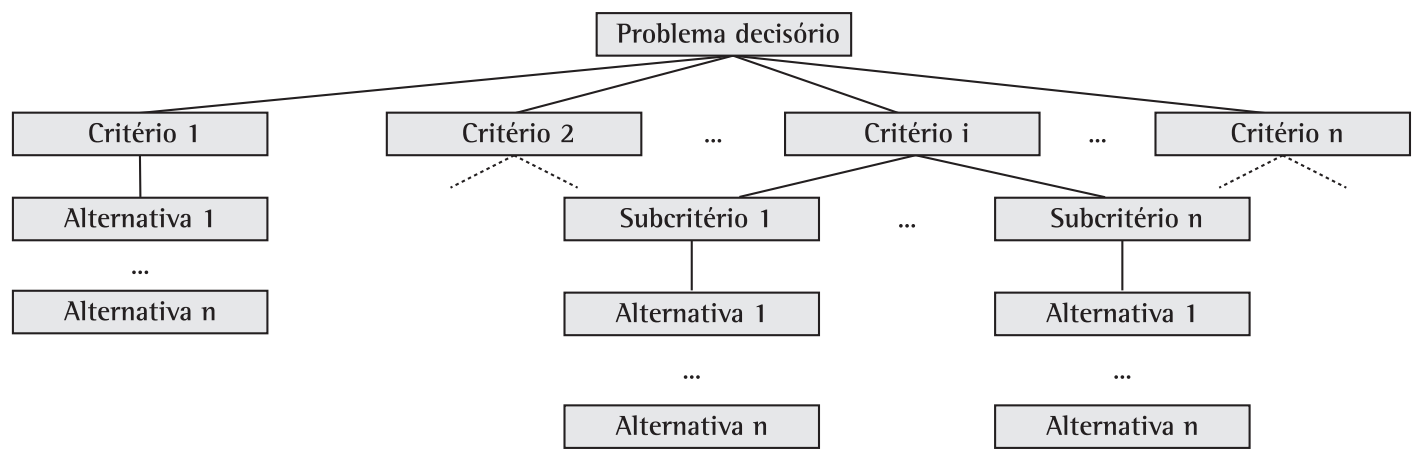

Figura 1. Árvore de critérios.

Tabela 1. Escala fundamental de Saaty.

\begin{tabular}{cl}
\hline Escala numérica & \multicolumn{1}{c}{ Escala verbal } \\
\hline 1 & Mesma importância \\
3 & Importância moderada de um sobre o outro \\
5 & Importância essencial ou forte \\
7 & Importância muito forte \\
9 & Importância extrema \\
$2,4,6,8$ & Valores intermediários \\
\hline
\end{tabular}

Antes de avançar na utilização do autovetor normalizado é preciso avaliar a consistência dos julgamentos armazenados nas matrizes de comparações paritárias. Essa avaliação é feita usando o índice de consistência (IC) da matriz. Para mais detalhes sobre os procedimentos para o cálculo do $\mathrm{IC}$ e seu significado, recomenda-se a leitura de Saaty (2006).

Depois de efetuar o preenchimento de todas as matrizes com os julgamentos dos especialistas e verificar os seus índices de consistência, é possível agregar todos os vetores de pesos através de uma função linear $f(a)$ aditiva que atribuirá a cada alternativa um valor final. As alternativas serão ordenadas e as que possuirem maior magnitude serão as preferiveis.

$f(a)=\sum_{j=1}^{n} w_{j} v_{j}(a)$

Onde:

- $w_{j}=$ peso do j-ésimo critério;

- $v_{j}(a)=$ desempenho da alternativa "a" com relação ao j-ésimo critério.

\section{Teoria dos Prospectos}

A Teoria dos Prospectos pertence ao campo da psicologia cognitiva e tem como base de seu paradigma a modelagem do comportamento do ser humano em face ao risco, no que diz respeito à tomada de decisões. 0 comportamento de decisores, observados pelos criadores da teoria, mostra que, nas situações

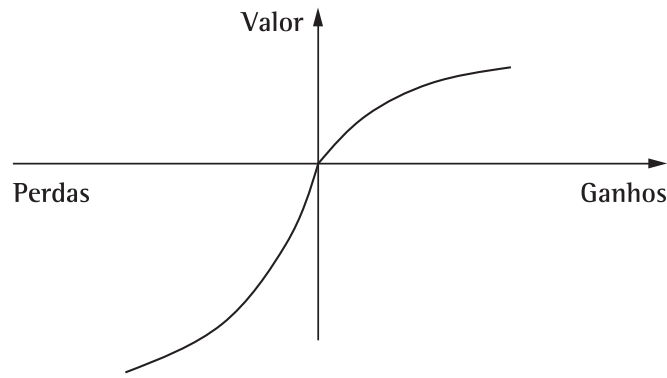

Figura 2. Função de valor da Teoria dos Prospectos.

que envolvem ganhos, seres humanos tendem a ser mais conservadores em relação ao risco e, em situações que envolvem perdas, mostram-se mais propensos ao risco. lsto é, quando se estabelece uma situação em que se pode ganhar, prefere-se um ganho menor, porém certo, a se arriscar por ganhos maiores e incertos. Em situações que envolvem perdas, as pessoas preferem se arriscar a perder mais, porém, com a possibilidade de nada perderem, a ter uma perda menor, porém, certa. Foi verificado esse comportamento dos seres humanos por meio de vários experimentos, aplicados a uma quantidade de pessoas considerada adequada.

0 método TODIM utiliza na sua fundamentação a Teoria dos Prospectos, incorporando a curva da função de valor determinada experimentalmente por Kahneman e Tversky (1979) ao seu modelo analítico. A curva experimental é exibida na Figura 2.

A partir da incorporação dessa função de valor, permite-se estabelecer uma medida quantitativa da satisfação das pessoas, inserindo ao modelo a característica de aversão e propensão ao risco, natural dos seres humanos.

\section{0 método TODIM}

0 método TODIM, sigla que significa Tomada de Decisão Interativa Multicritério, é um método 
multicritério de análise de decisão, desenvolvido pelo professor brasileiro Luiz Flávio Autran Monteiro Gomes. Após sua aplicação, são obtidas, como resultado, as altenativas já sequenciadas por ordem de preferência. 0 método utiliza modelagem matemática baseada em matrizes de comparações por pares como no AHP, porém, incorpora em seu modelo a Teoria dos Prospectos de Kahneman e Tversky (1979), na qual se descreve o comportamento do ser humano em face ao risco, conforme mencionado na seção 3. Existem vários textos que descrevem esse método. $\mathrm{Na}$ literatura, encontram-se: livro (GOMES; ARAYA; CARIGNANO, 2004), dissertações de mestrado (PASSOS, 2002; MORAES, 1999) e artigos (GOMES; LIMA, 1992; GOMES; RANGEL, 2009).

Algumas pré-condições tornam-se necessárias antes que o método possa ser aplicado. Antes de iniciar a construção do modelo, é necessário que os critérios sejam bem selecionados atendendo ao pré-requisito de separabilidade, de forma que critérios não sejam contabilizados mais de uma vez dentro do mesmo modelo (CLEMEN, 1996). 0 método TODIM baseia-se também na Teoria da Utilidade Aditiva e, para que haja separabilidade entre critérios, estes devem ser mutuamente preferencialmente independentes. Um atributo $Y$ é dito preferencialmente independente do atributo $X$ se as preferências para resultados específicos de $Y$ não dependerem do nível do atributo $X$, e vice-versa.

Após a seleção dos critérios e alternativas, montam-se duas matrizes. A primeira é a matriz de julgamentos, que possui $\mathrm{n}$ (número de alternativas) linhas e $m$ (número de critérios) colunas. É realizada então uma consulta a pessoas (especialistas) capazes de opinar sobre a importância relativa das diversas alternativas, critérios e subcritérios do problema, sempre a partir de sua representação através da hierarquia. Estes decisores procedem com a valoração para cada uma das alternativas, critérios e subcritérios, e estes valores são inseridos na matriz.

Para o clássico exemplo da escolha de um automóvel, valores quantitativos (p.ex., consumo de combustível) são facilmente inseridos nas colunas. Já para julgamentos subjetivos (p.ex., conforto interno), são designados valores por meio da leitura da Tabela 2 (GOMES; LIMA, 1992) que relaciona estes julgamentos através de uma escala numérica. Posteriormente, faz-se a normalização através da divisão de cada coluna pelo seu maior valor.

A segunda matriz é a de comparação entre pares de critérios. Nessa matriz, comparam-se os critérios entre si da mesma forma como se faz com o método AHP.

0 método TODIM fornece como resultado final o valor global das alternativas sequenciadas por ordem de preferência. Para que se façam os cálculos dos valores de cada alternativa, é necessário que, antes, sejam determinadas as dominâncias de cada alternativa em relação a cada uma das outras. A incorporação do paradigma da Teoria dos Prospectos pelo método TODIM se faz pela introdução dessa função de valor nas medidas de dominância de uma alternativa sobre a outra. Em um contexto multicritério, as perdas e os ganhos são definidos como diferenças entre os valores $w$ estabelecidos na matriz de julgamentos, para todas as alternativas, dentro de um critério $c$ em particular. As equações constitutivas do método, portanto, são:

$$
\delta(i, j)=\sum_{c=1}^{m} \Phi_{c}(i, j), \quad \forall(i, j)
$$

Em que

$$
\Phi_{c}(i, j)=\left\{\begin{array}{l}
\sqrt{\frac{a_{r c}\left(w_{i c}-w_{j c}\right)}{\sum_{c} a_{r c}}, \text { se } w_{i c}-w_{j c}>0} \\
0, \text { se } w_{i c}-w_{j c}=0 \\
-\frac{1}{\theta} \sqrt{\frac{\left(\sum_{c} a_{r c}\right)\left(w_{j c}-w_{i c}\right)}{a_{r c}}}, \text { se } w_{i c}-w_{j c}<0
\end{array}\right.
$$

e

- $\delta(i, j)=$ medida de dominância da alternativa $i$ sobre a alternativa $j$;

- $m$ = número de critérios;

- $c$ = critério genérico variando de 1 a $m$;

- $a_{r c}=$ taxa de substituição do critério $c$ pelo critério $r$ (elemento da matriz de comparação por pares de critérios);

- $w_{i c}, w_{j c}=$ pesos das alternativas $i \mathrm{e} j$, respectivamente, em relação ao critério $c$;

- $\theta=$ fator de atenuação de perdas.

Na prática, a função de valor incorporada ao TODIM se torna proporcional à raiz quadrada da diferença entre os valores $w$. Isso significa que, dado o aumento da diferença, maior será o valor da função $\Phi(i, j)$, porém ocorrerá em taxas decrescentes. lsso também é o que ocorre quando se interpreta o conceito da função de valor da Teoria dos Prospectos e indica a aversão ao risco.

Após serem efetuados os cálculos, será montada a matriz quadrada $\delta(i, j)$, de ordem $\mathrm{n}$, onde $\mathrm{n}$ é o número de alternativas. Esta matriz é denominada matriz de dominâncias relativas das alternativas. Os valores totais das alternativas são determinados através do seguinte cálculo:

$$
\xi_{i}=\frac{\sum_{j=1}^{n} \delta(i, j)-\operatorname{Min}_{i} \sum_{j=1}^{n} \delta(i, j)}{\operatorname{Max}_{i} \sum_{j=1}^{n} \delta(i, j)-\operatorname{Min}_{i} \sum_{j=1}^{n} \delta(i, j)}
$$


Tabela 2. Substituição da escala verbal pela escala cardinal.

\begin{tabular}{|c|c|c|c|}
\hline $\begin{array}{l}\text { Intensidade de } \\
\text { importância }\end{array}$ & Definição & $\begin{array}{l}\text { Comparação } \\
\text { alternativa } \mathrm{x} \text { critério }\end{array}$ & $\begin{array}{l}\text { Comparação } \\
\text { critério x critério }\end{array}$ \\
\hline 0 & $\begin{array}{l}\text { Nenhuma } \\
\text { importância }\end{array}$ & $\begin{array}{c}\text { A alternativa } i \text { não contribui } \\
\text { para o critério } c\end{array}$ & - \\
\hline 1 & - & $\begin{array}{l}\text { A alternativa } i \text { tem muito pequena } \\
\text { importância para o critério } c\end{array}$ & $\begin{array}{c}0 \text { critério } p \text { tem importância igual } \\
\text { ao critério } q\end{array}$ \\
\hline 2 & - & $\begin{array}{l}\text { A importância da alternativa } i \text { está entre } \\
\text { muito pequena e pequena para o critério } c\end{array}$ & $\begin{array}{l}\text { A importância do critério } p \text { está entre igual e } \\
\text { ligeiramente maior do que a do critério } q\end{array}$ \\
\hline 3 & $\begin{array}{l}\text { Pequena } \\
\text { importância }\end{array}$ & $\begin{array}{l}\text { A alternativa } i \text { tem pequena } \\
\text { importância para o critério } c\end{array}$ & $\begin{array}{l}0 \text { critério } p \text { é ligeiramente mais } \\
\text { forte do que o critério } q\end{array}$ \\
\hline 4 & Importante & $\begin{array}{l}\text { A alternativa } i \text { tem alguma } \\
\text { importância para o critério } c\end{array}$ & $\begin{array}{l}0 \text { critério } p \text { é mais importante } \\
\text { do que o critério } q\end{array}$ \\
\hline 5 & Forte importância & $\begin{array}{l}\text { A alternativa } i \text { tem forte importância } \\
\text { para o critério } c\end{array}$ & $\begin{array}{l}0 \text { critério } p \text { é fortemente mais } \\
\text { importante do que o critério } q\end{array}$ \\
\hline 6 & - & $\begin{array}{l}\text { A importância da alternativa } i \text { está entre } \\
\text { forte e bastante forte para o critério } c\end{array}$ & $\begin{array}{c}\text { A importância do critério } p \text { está entre } \\
\text { forte e muito fortemente maior do } \\
\text { que a do critério } q\end{array}$ \\
\hline 7 & $\begin{array}{l}\text { Muito forte } \\
\text { importância }\end{array}$ & $\begin{array}{l}\text { A alternativa } i \text { tem muito forte } \\
\text { importância para o critério } c\end{array}$ & $\begin{array}{l}0 \text { critério } p \text { é muito forte/mais } \\
\text { importante do que o critério } q\end{array}$ \\
\hline 8 & - & $\begin{array}{l}\text { A importância da alternativa } i \text { está entre } \\
\text { muito forte e absoluta para o critério } c\end{array}$ & $\begin{array}{c}\text { A importância do critério } p \text { está entre muito } \\
\text { fortemente e absolutamente maior } \\
\text { do que a do critério } q\end{array}$ \\
\hline 9 & $\begin{array}{c}\text { Absoluta } \\
\text { importância }\end{array}$ & $\begin{array}{l}\text { A alternativa } i \text { tem absoluta } \\
\text { importância para o critério } c\end{array}$ & $\begin{array}{l}0 \text { critério c é absolutamente mais } \\
\text { importante do que o critério } q\end{array}$ \\
\hline
\end{tabular}

Cada valor $\xi$ é uma soma de linhas normalizadas da matriz de dominâncias. Depois de calculados os valores, estes são ordenados e, assim, determinam-se as alternativas a serem escolhidas.

\section{0 estudo de caso}

Nesta seção é mostrado o estudo de caso para a listagem da ordem de prioridade das tecnologias em um sistema tático de comunicações. Portanto, são resolvidos dois problemas: um para subsistema de dados e outro para o de combate. Como indicado na Tabela 3, há 5 princípios gerais ao emprego das comunicações militares, isto é, os sistemas alternativos precisam atender a alguns critérios. Salles et al. (2008) descrevem essas grandezas/ critérios como:

- Usabilidade: facilidade de utilização e configuração dos elementos da rede; deve estar diretamente relacionado ao nível de automação;

- Desempenho: valores associados a critérios como atraso, jitter (variação de atraso), acurácia, disponibilidade, redundância e prioridade;

- Escalabilidade: a capacidade de um sistema expandir-se sem degradar-se. Quando os parâmetros relacionados ao desempenho da rede permanecem inalterados, mesmo com a ativivação de todos os componentes possíveis de uma rede, diz-se que o grau de escalabilidade é satisfatório;

- Gerenciabilidade: capacidade de levantarem-se parâmetros da rede para utilização deles como base para ações preventivas. É essencial a existência de
Tabela 3. Princípios gerais de emprego das comunicações do Exército Brasileiro (SALLES et al., 2008).

\begin{tabular}{ccc}
\hline $\begin{array}{c}\text { Principios } \\
\text { gerais }\end{array}$ & $\begin{array}{c}\text { Princípio de emprego das } \\
\text { comunicações militares }\end{array}$ & $\begin{array}{c}\text { Percentual } \\
(\%)\end{array}$ \\
\hline Escalabilidade & $\begin{array}{c}\text { Amplitude de desdobramento, } \\
\text { integração }\end{array}$ & 37,5 \\
Desempenho & $\begin{array}{c}\text { Tempo integral, rapidez, confiabilidade, } \\
\text { continuidade, prioridade } \\
\text { Segurança }\end{array}$ & 31,1 \\
Gerenciabilidade & $\begin{array}{c}\text { Apoio em profundidade, emprego } \\
\text { centralizado, apoio cerrado }\end{array}$ & 14,9 \\
Usabilidade & Flexibilidade & 5,7 \\
\hline
\end{tabular}

uma arquitetura gerencial cooperativa, o estudo contínuo da composição do tráfego nas diversas linhas, controle no uso de enlaces e visualização multidimensional da rede;

- Segurança: proteção dos recursos, com uso de senhas, criptografias, controle rígido de usuários.

A partir da Tabela 3 de princípios gerais, os autores classificam os requisitos dos subsistemas de Rádio Combate e de Dados, nas quais serviram de base para a construção das árvores de critérios. As Figuras 3 e 4 mostram a árvore dos subsistemas de Rádio Combate e de Dados, respectivamente. Subcritérios são utilizados com o intuito de tornar mais concreto o significado de cada grandeza dos princípios gerais.

Os subsistemas de Rádio de Combate e de Dados se diferenciam por seus subcritérios. Abaixo é apresentada a descrição de cada subcritério, concedida nas entrevistas realizadas com os autores do artigo base: 


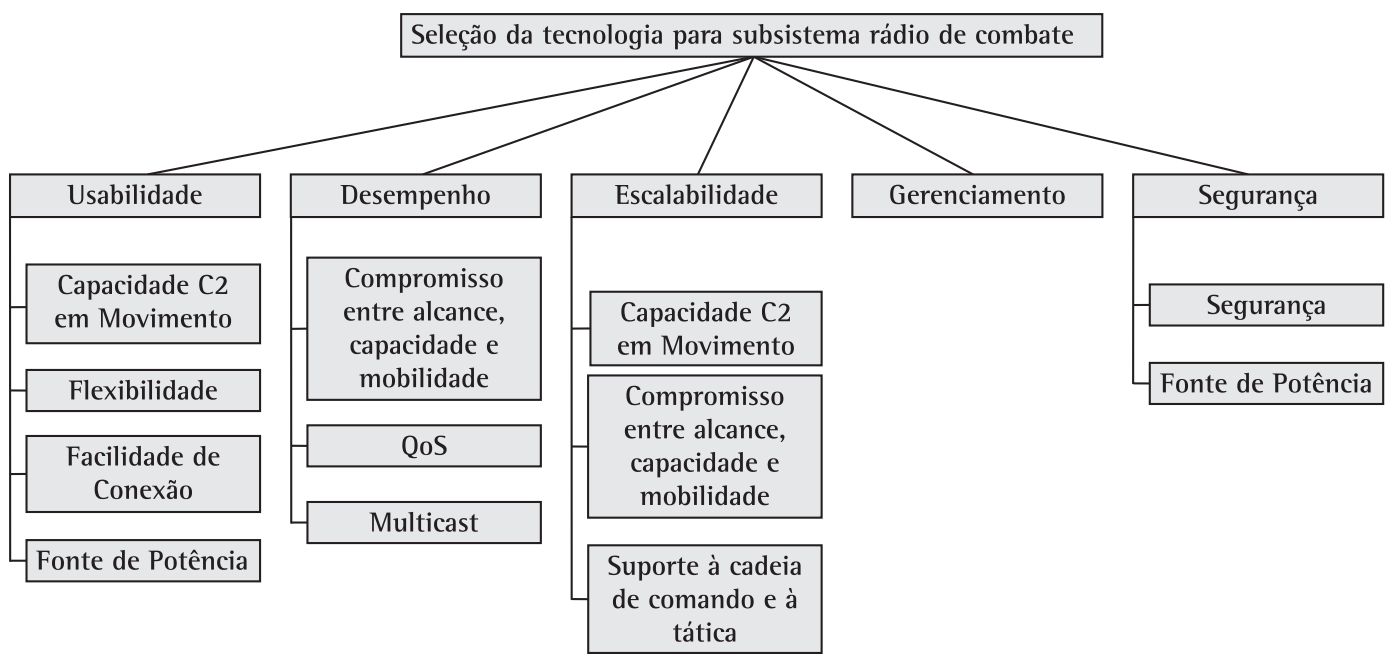

Figura 3. Árvore de critérios do subsistema de Rádio de Combate.

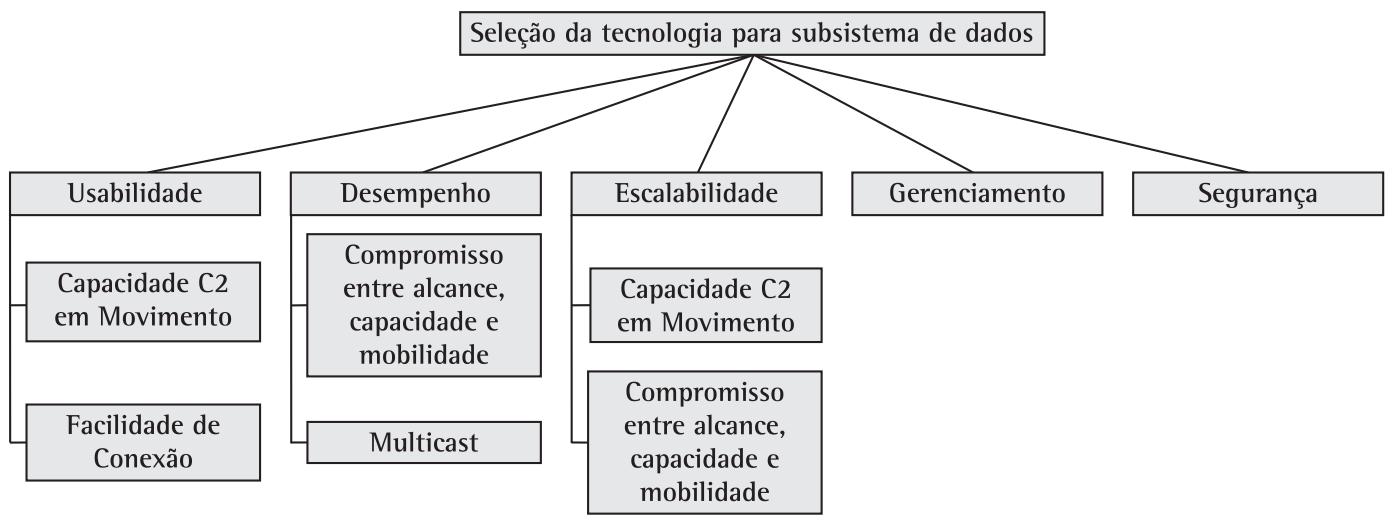

Figura 4. Árvore de critérios do subsistema de Dados.

- Capacidade C2 em movimento: tecnologia capacitada às atividades de Comando e Controle em movimento;

- Flexibilidade: capacidade do sistema executar uma operação em quaisquer condições de terreno e tempo;

- Facilidade de Conexão: facilidade de implantar e trabalhar com a tecnologia;

- Fonte de Potência: quanto menor a potência necessária para um sistema funcionar, ele é mais bem classificado. lsso se deve por motivos de segurança, uma vez que dificulta a detecção do sistema pelos inimigos;

- Compromisso entre alcance, mobilidade e capacidade: o sistema deve oferecer suporte que atenda ao mesmo tempo: o alcance necessário da rede, a mobilidade dos usuários e a capacidade de transmissão do sinal;

- QoS: qualidade do serviços de comunicação em tempo real oferecido pela tecnologia;
- Multicast: sistema que realiza a entrega de informação para múltiplos destinatários simultaneamente, usando estratégias eficientes;

- Suporte à cadeia de comando e à tática: sistema não apresenta restrições a localizações de frações ou elementos, tem capacidade de alteração de composição de frações QoS e dá suporte aos dados críticos quanto ao tempo (prioridade e precedência).

Foram analisados casos de redundâncias entre critérios nas duas árvores, mas este problema não foi encontrado. Portanto, nenhum critério proposto no artigo foi removido. 0 subcritério Capacidade C2 em movimento possui conotações diferentes quando associado ao critério Usabilidade e Escalabilidade. Da mesma forma o subcritério Compromisso entre Alcance, Capacidade e Mobilidade que possui diferentes interpretações sob a óptica dos critérios escalabilidade e desempenho. 


\subsection{Alternativas}

Nesta seção, são apresentadas as tecnologias descritas em Salles et al. (2008) quanto ao possível emprego em Sistemas de comunicação do Exército Brasileiro.

- Rede Rádio de Combate: utiliza uma rede rádio legada para transmissão de dados. Pode trabalhar em algumas faixas de transmissão (HF, VHF ou UHF);

- Rede Pacote Rádio: interliga diversas subredes por máquinas bridges, utilizando a mesma estrutura convencional das Redes Rádio de Combate;

- Redes Ad-Hoc: não necessitam de nenhuma preparação da área de cobertura porque elas proveem comunicação sem itens como torres ou linhas de transmissão. Os pontos-chave dessas redes são o controle de acesso ao meio, utilizado para compartilhar os recursos de canal entre os nós e o roteamento, que consiste em encontrar uma rota entre emissor e receptor através de um número desconhecido de nós intermediários;

- TDMA Repetido: cada estação instalada transmite em um slot de tempo próprio, o qual é determinado por uma estação de controle. Alcançam taxas de transmissão de aproximadamente $500 \mathrm{kbps}$;

- Redes de Arquitetura Estação-Base: as redes fazem uso de uma estação base para intermediar a comunicação entre quaisquer duas estações, como nos sistemas de telefonia celular e de rádios half-duplex de frequência dupla;

- Redes Rádio UWB: atinge altas velocidades de transmissão de dados e pequeno alcance. Isso se deve pela utilização de sinais de rádios de baixa energia na fonte de pulsos curtos (0.1 a $1.5 \mathrm{~ns}$ ).

\subsection{Abordagem com o AHP}

A utilização do método AHP Clássico pressupõe a utilização de matrizes de comparações paritárias que servirão para analisar a importância relativa entre os critérios e subcritérios (tipo 1), assim como atribuir valores relativos quantitativos que indiquem a ordenação das alternativas à luz de determinado critério ou subcritério (tipo 2). Visto isso, será necessário preencher 4 matrizes tipo 1 e 13 matrizes tipo 2, somente para o Subsistema Rádio de Combate. A rigor também seria necessário coletar julgamentos para os critérios pai. Mas como essas importâncias relativas são dados iniciais da modelagem, descritos na Tabela 3, esse trabalho não será necessário. Para o Subsistema Rádio de Dados será necessário preencher 3 (três) matrizes tipo 1 e 8 (oito) matrizes tipo 2 . A implementação do AHP pode ser feita facilmente utilizando planilhas eletrônicas.
Para ilustrar esse trabalho, serão mostrados os julgamentos de especialistas na área, refletidos no preenchimento de uma matriz tipo 1 e outra tipo 2 para o subsistema de Rádio de Combate. Para o critério usabilidade, foram extraídos os dados constantes na Tabela 4 preenchida com valores da escala fundamental de Saaty (Tabela 1).

A Tabela 4 depois de ser normalizada pela soma dos elementos de cada coluna apresentará os resultados da Tabela 5. No caso da matriz de usabilidade o índice de consistência é $1 \mathrm{C}=0,0145$

Após efetuar a média aritmética das linhas e normalizar os valores, foi obtido o vetor de pesos para a Tabela 5. Esse vetor está representado na Tabela 6 .

Como resultado final, foi possível estabelecer as pontuações para as alternativas conforme descrito na Tabela 7.

Tabela 4. Matriz de comparação dos subcritérios de usabilidade.

\begin{tabular}{|c|c|c|c|c|}
\hline & $\begin{array}{c}\text { Capacidade } \\
\text { C2 em } \\
\text { movimento }\end{array}$ & Flexibilidade & $\begin{array}{c}\text { Facilidade } \\
\text { de } \\
\text { conexão }\end{array}$ & $\begin{array}{c}\text { Fonte } \\
\text { de } \\
\text { potência }\end{array}$ \\
\hline $\begin{array}{l}\text { Capacidade } \\
\text { C2 em } \\
\text { movimento }\end{array}$ & 1 & 3 & 3 & 5 \\
\hline Flexibilidade & $1 / 3$ & 1 & 1 & 3 \\
\hline $\begin{array}{l}\text { Facilidade de } \\
\text { conexão }\end{array}$ & $1 / 3$ & 1 & 1 & 3 \\
\hline $\begin{array}{l}\text { Fonte de } \\
\text { potência }\end{array}$ & $1 / 5$ & $1 / 3$ & $1 / 3$ & 1 \\
\hline Soma & 1,87 & 5,33 & 5,33 & 12,00 \\
\hline
\end{tabular}

Tabela 5. Matriz dos subcritérios de usabilidade normalizada.

\begin{tabular}{ccccc}
\hline & $\begin{array}{c}\text { Capacidade } \\
\text { C2 em } \\
\text { movimento }\end{array}$ & Flexibilidade & $\begin{array}{c}\text { Facilidade } \\
\text { de } \\
\text { conexão }\end{array}$ & $\begin{array}{c}\text { Fonte } \\
\text { de } \\
\text { potência }\end{array}$ \\
\hline $\begin{array}{c}\text { Capacidade } \\
\text { C2 em } \\
\text { movimento }\end{array}$ & 0,5357 & 0,5625 & 0,5625 & 0,4167 \\
$\begin{array}{c}\text { Flexibilidade } \\
\text { Facilidade de } \\
\text { conexão }\end{array}$ & 0,1786 & 0,1875 & 0,1875 & 0,2500 \\
$\begin{array}{c}\text { Fonte de } \\
\text { potência }\end{array}$ & 0,1071 & 0,0625 & 0,0625 & 0,0833 \\
\hline
\end{tabular}

Tabela 6. Vetor de pesos.

\begin{tabular}{cc}
\hline Subcritério & Peso \\
\hline Capacidade C2 em movimento & 0,5193 \\
Flexibilidade & 0,2009 \\
Facilidade de conexão & 0,2009 \\
Fonte de potência & 0,0789 \\
\hline
\end{tabular}


Tabela 7. Resultado final da análise com o AHP para os dois problemas.

\begin{tabular}{cccccc}
\hline & \multicolumn{2}{c}{ Subsistema de combate } & & \multicolumn{2}{c}{ Subsistema de dados } \\
\cline { 2 - 3 } \cline { 5 - 6 } & Pontuação & Ordenação & & Pontuação & Ordenação \\
\hline Rede Rádio de Combate com Suporte a Dados & 10,3217 & $5^{\circ}$ & & 9,4388 & $6^{\circ}$ \\
Rede de Rádio de Pacotes & 16,3409 & $3^{\circ}$ & & 13,6423 & $5^{\circ}$ \\
Rede Ad Hoc & 29,0190 & $1^{\circ}$ & & 16,0104 & $3^{\circ}$ \\
TDMA Repetido & 14,3279 & $4^{\circ}$ & & 23,5476 & $1^{\circ}$ \\
Arquitetura Base-Estação & 8,7422 & $6^{\circ}$ & & 14,0398 & $4^{\circ}$ \\
UWB & 18,6318 & $2^{\circ}$ & & 21,3241 & $2^{\circ}$ \\
\hline
\end{tabular}

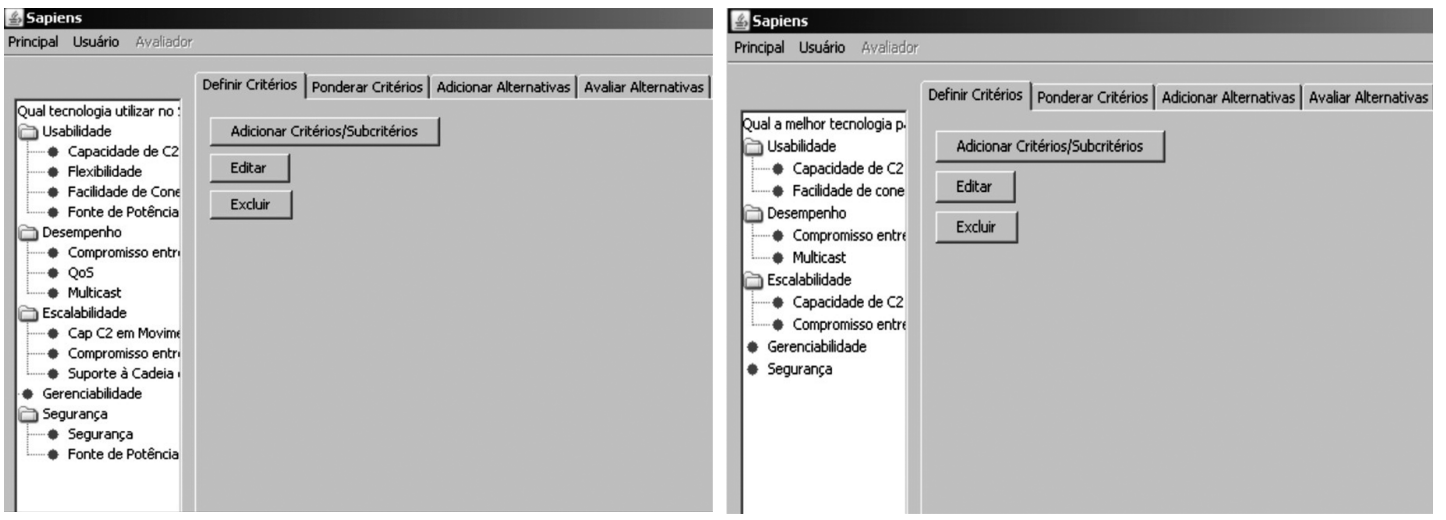

Figura 5. Critérios de análise dos subsistemas Rádio de Combate e de Dados.

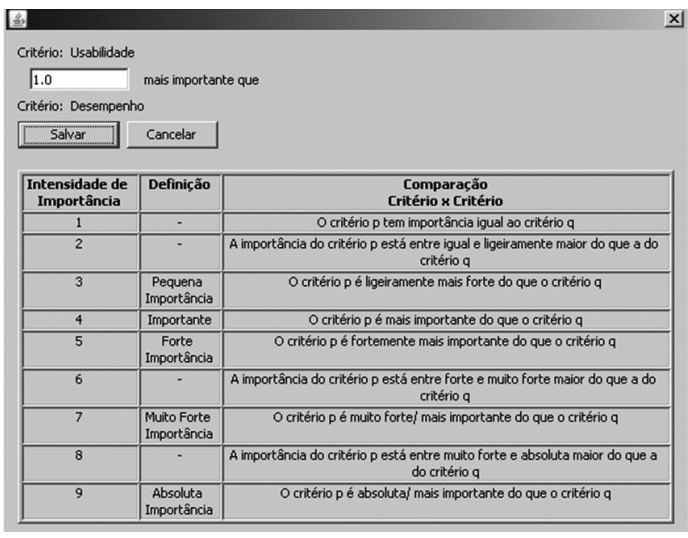

Figura 6. Comparação dos critérios pelo Sapiens.

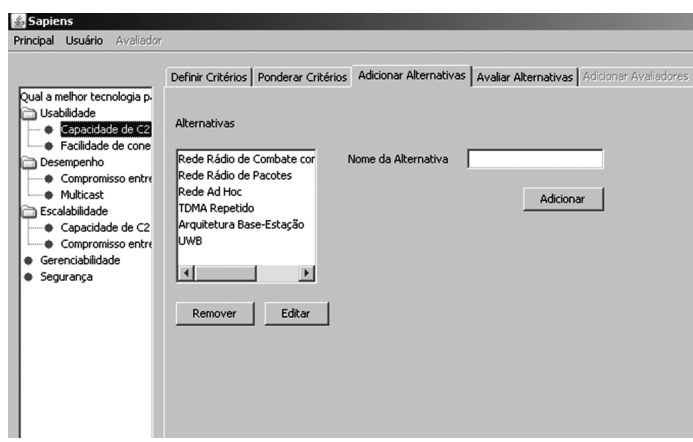

Figura 7. Entrada das alternativas do problema no Sapiens.
Tabela 8. Resultado final da análise com o TODIM para os dois problemas.

\begin{tabular}{ccc}
\hline Posição & $\begin{array}{c}\text { Subsistema Rádio } \\
\text { de Combate }\end{array}$ & $\begin{array}{c}\text { Subsistema Rádio } \\
\text { de Dados }\end{array}$ \\
\hline $1^{\text {a }}$ & Redes Ad Hoc & UWB \\
$2^{\text {a }}$ & UWB & TDMA repetido \\
$3^{\text {a }}$ & Redes Rádio de Pacotes & Redes Ad Hoc \\
$4^{\text {a }}$ & TDMA repetido & Redes Rádio de Pacotes \\
$5^{\text {a }}$ & Rede Rádio de Combate & Arquitetura Base-Estação \\
$6^{\text {a }}$ & Arquitetura Base-Estação & Rede Rádio de Combate \\
\hline
\end{tabular}

\subsection{Abordagem com o TODIM}

0 software Sapiens foi empregado em todo o processo de modelagem multicritério. 0 usuário interessado em baixar o software poderá fazê-lo no endereço eletrônico: www.comp.ime.eb.br/ todima. Esse endereço trás informações sobre o Projeto TODIMA (Tomada de Decisão Interativa Multicritério Automatizada) e, lá, é possível encontrar um link para download do software Sapiens. A grande vantagem de utilizar o software é poder inserir os julgamentos e obter os resultados de maneira muito mais rápida do que da forma convencional, utilizando planilhas eletrônicas. Vista a razoável complexidade das equações do TODIM e a grande possibilidade de erros inerentes à utilização de planilhas, sem dúvida ter um software disponível contribuirá bastante para os interessados em trabalhar com o TODIM. 
Tabela 9. Comparação dos resultados finais com o AHP e o TODIM.

\begin{tabular}{|c|c|c|c|c|}
\hline & \multicolumn{2}{|c|}{ Subsistema de Combate } & \multicolumn{2}{|c|}{ Subsistema de Dados } \\
\hline & AHP & TODIM & AHP & TODIM \\
\hline 1 & Redes Ad Hoc & Redes Ad Hoc & TDMA Repetido & UWB \\
\hline 2 & UWB & UWB & UWB & TDMA Repetido \\
\hline 3 & Rede de Rádio de Pacotes & Rede de Rádio de Pacotes & Redes Ad Hoc & Redes Ad Hoc \\
\hline 4 & TDMA Repetido & TDMA Repetido & Arquitetura Base-Estação & Rede de Rádio de Pacotes \\
\hline 5 & $\begin{array}{l}\text { Rede Rádio de Combate } \\
\text { com Suporte a Dados }\end{array}$ & $\begin{array}{l}\text { Rede Rádio de Combate } \\
\text { com Suporte a Dados }\end{array}$ & Rede de Rádio de Pacotes & Arquitetura Base-Estação \\
\hline 6 & Arquitetura Base-Estação & Arquitetura Base-Estação & $\begin{array}{l}\text { Rede Rádio de Combate } \\
\text { com Suporte a Dados }\end{array}$ & $\begin{array}{l}\text { Rede Rádio de Combate } \\
\text { com Suporte a Dados }\end{array}$ \\
\hline
\end{tabular}

A árvore de critérios para cada problema foi inserida na coluna da direita da interface, conforme descrito na Figura 5.

Após inserir a árvore de critérios, o próximo passo é atribuir pesos relativos aos critérios. Esses pesos são atribuídos através de matrizes de comparações paritárias conforme é feito no AHP. Dessa maneira, os mesmos julgamentos realizados anteriormente podem ser aqui aproveitados. 0 usuário do software que for inserir informações terá disponível nesse momento uma reprodução da Tabela 2 na tela do computador, conforme descrito na Figura 6.

0 passo seguinte à atribuição dos pesos relativos entre os critérios é identificar quais são as alternativas disponíveis para posteriormente atribuir valores para essas alternativas à luz dos critérios já estabelecidos. Essa fase está ilustrada na Figura 7.

Para obter os resultados finais bastará ao usuário selecionar a aba "resultado". Isso fornecerá as alternativas pontuadas entre 0 (zero) e 1 (um), a preferivel terá valor 1 (um) e as menos preferiveis terão outros valores intermediários, decrescendo até chegar no valor 0 (zero). 0 resultado final dessa modelagem está descrito na Tabela 8.

\section{Conclusão e análise de resultados}

Esse trabalho mostrou a estruturação de um problema de seleção de equipamentos comum na área militar. A grande vantagem na utilização da abordagem multicritério é trazer maior transparência e clareza ao processo decisório e na obtenção do resultado final. Um problema como esse, cuja análise envolve aspectos extremamente técnicos e necessidade de vivência no meio das comunicações militares, sem dúvida se beneficia dos procedimentos construtivos e raciocínio estruturado próprios da análise de decisão multicritério.

É importante ressaltar que as ordenações aqui obtidas necessitam ainda da realização de criteriosa análise de sensibilidade, que revise e confirme os julgamentos fornecidos para a obtenção dos resultados.
Parece ser bem intuitivo, inclusive para os leigos, que alguns resultados da Tabela 2 deveriam ser revistos. Um exemplo claro é o critério "segurança" que lá representa somente 14,9\% de importância dentro do conjunto principal de critérios. Isso é completamente incoerente dentro do meio militar. Entretanto, os resultados aqui descritos na Tabela 2 refletem as informações compiladas nos manuais de militares de campanha. Assim, ou os manuais precisariam ser rediscutidos, ou um grupo de reconhecidos especialistas na área deveria se reunir para criar a modelagem mais adequada para eles.

O software Sapiens, desenvolvido dentro do Instituto Militar de Engenharia é uma primeira versão bastante simples que implementa o método TODIM. Mas, apesar da sua simplicidade, ele atende perfeitamente às necessidades do usuário que deseja trabalhar com o método. Já está sendo desenvolvido o projeto da próxima versão, mais completa, com mais recursos e capaz de modelar problemas multicritério, não só de ordenação, mas também de classificação. Serão incluídos, também, mais recursos gráficos, aprimoramentos da interface, recursos para análise de sensibilidade e decisão em grupo.

É importante fazer a comparação com os resultados obtidos com o AHP e o TODIM. A Tabela 9 auxiliará na visão geral dos resultados.

Para o subsistema Rádio de Combate, a ordenação com ambos os métodos foi idêntica. Para o subsistema rádio de dados, houve uma inversão entre o primeiro e o segundo colocados e outras diversas alterações. A única coincidência foi no posicionamento das redes Ad hoc na terceira posição. Como não houve um processo de análise de sensibilidade cauteloso nessa avaliação, se torna mais difícil fazer julgamentos mais detalhados. Entretanto, é válido observar que as pontuações, em ambos os métodos, para as alternativas TDMA repetido e UWB são muito próximas. Vale ressaltar que as equações matemáticas utilizadas pelas metodologias são bem diferentes. No AHP a agregação dos julgamentos é feita utilizando uma equação linear aditiva, conforme mostrado na seção 2 . No TODIM, a agregação dos julgamentos é feita com as equações 
condicionais mostradas na seção 4, estruturadas sobre o paradigma da Teoria dos Prospectos. Por isso, a existência de julgamentos muito próximos para duas alternativas pode fazer com que haja a inversão de posicionamento nos resultados quando ambas as metodologias são comparadas.

0 AHP possui vários softwares que o implementam. 0 mais popular é o Expert Choice, que é um software proprietário.

A utilização de ambos os métodos é adequada para esse tipo de problema. Entretanto, o método TODIM possui algumas vantagens. A primeira é a menor quantidade de julgamentos que são necessários para a obtenção dos mesmos resultados. Na fase de atribuição de valores às alternativas com relação aos critérios, com o TODIM, são feitos $n \times c$ julgamentos (onde $\mathrm{n}$ é o número de alternativas e c é o número de critérios). Com o AHP são feitos $\frac{n(n-1)}{2} \times c$ julgamentos. Isso torna o AHP muito mais cansativo para o decisor, podendo levar a erros por fadiga, principalmente quando cresce o número de alternativas. O AHP clássico possui uma série de críticas sérias sobre a sua formulação. Entretanto, modificações simples podem fazer com que essas críticas sejam contornadas. Para saber mais sobre essas críticas, é sugerida a leitura de Gomes (2007) e Goodwin e Wright (2000). A principal desvantagem do método TODIM é a relativa complexidade matemática de suas equações quando comparado a outras metodologias multicritério. Entretanto, com a utilização de um software, essas equações ficam escondidas e a obtenção de uma solução fica simples e muito mais rápida.

\section{Referências}

BRASIL. Estado-Maior do Exército. C 11 - 61: comunicações na divisão de Exército. Brasília, DF, 1995.

BRASIL. Estado-Maior do Exército. C 11 - 30: as comunicações na Brigada. Brasília, DF, 1998.

CLEMEN, R. T. Making hard decisions: an introduction to decision analysis. Duxbury Press, 1996. 664 p.
GOMES, L. F. A. M. Teoria da decisão. São Paulo: Thomson, 2007. $116 \mathrm{p}$.

GOMES, L. F. A. M.; ARAYA, M. C. G.; CARIGNANO, C. Tomada de decisões em cenários complexos. São Paulo: Thomson, 2004. $168 \mathrm{p}$.

GOMES, L. F. A. M.; LIMA, M. P. P. From modelling individual preferences to multicriteria ranking of discrete alternatives: a look at prospect theory and the additive difference model. Foundations of Computing and Decision Sciences, v. 17, n. 3, 1992.

GOMES, L. F. A. M.; RANGEL, L. A. D. An application of the TODIM method to the multicriteria rental evaluation of residential properties. European Journal of Operational Research, v. 193, p. 204-211, 2009. http://dx.doi. org/10.1016/j.ejor.2007.10.046

GOODWIN, P.; WRIGHT, G. Decision analysis for management judgment. Chichester: John Wiley \& Sons, 2000. $454 \mathrm{p}$.

KAHNEMAN, D.; TVERSKY, A. Prospect theory: an analysis of decision under risk. Econometrica, v. 47, n. 2, p. 263-292, 1979. http://dx.doi.org/10.2307/1914185

MORAES, L. F. R. Avaliação multicritério de projetos de produção da indústria de petróleo no Brasil. Dissertação (Mestrado em Engenharia de Produção)-Departamento de Engenharia de Produção, Universidade Federal Fluminense, Niterói, 1999.

PASSOS, A. C. Avaliação multicritério de material de emprego militar. 2002. 79 f. Dissertação (Mestrado em Administração de Empresas)-Faculdades lbmec, Rio de Janeiro, 2002.

SAATY, T. L. Fundamentals of decision making and priority theory with the analytic hierarchy process. Pittsburgh: RWS publications, 2006. $478 \mathrm{p}$.

SALLES, R. M. et al. Novas perspectivas tecnológicas para o emprego das comunicações no Exército Brasileiro. Revista Militar de Ciência e Tecnologia, v. 25, p. 68-79, 2008.

\section{Agradecimentos}

Os autores agradecem ao engenheiro militar David Fernandes Cruz Moura, professor do IME, a valiosa contribuição no desenvolvimento da aplicação desenvolvida neste artigo e o apoio na montagem do software Sapiens nos servidores do IME. Agredecem também ao engenheiro militar Anderson Fernandes Pereira dos Santos, professor do IME, o seu apoio no aprimoramento do software Sapiens.

\title{
Selection of communication technologies in the Brazilian Army using AHP, TODIM and Sapiens software
}

\begin{abstract}
The communication networks in the Brazilian Army have special characteristics. A tactical system of communication should have a unique logical structure integrating multiple technologies. These logical structures for tactical activities are named, in Brazil, Combat Radio Subsystem and Data Radio Subsystem - each one with its specific purposes. There are some alternatives regarding the combat manuals requirements. This paper developed a multi-criteria ranking case study where the main matter was to choose the best technology for each subsystem. Two methodologies were employed: Analytic Hierarchy Process (AHP) and TODIM. A decision support software (based on TODIM) called Sapiens was used to implement the results for this method. The main objective of this paper was to build a framework for this kind of ranking problem.
\end{abstract}

Keywords

Multi-criteria decision analysis. TODIM. AHP. Military Communications. Sapiens software. 